Andreas

Wömpener

\section{Behavioral Budgeting - Beschränkte Rationalität von kognitiven Urteils- und Entscheidungsprozessen im Kontext der Budgetierung Dissertation}

\author{
Verlag Dr. Kovač \\ Hamburg 2008 \\ 270 Seiten, $88,00 €$ \\ ISBN: 978-3-8300-3857-3
}

Wer denkt, dass Management oder die Unternehmenssteuerung rein rationale oder ökonomische Prozesse umfassen, irrt. Nicht zuletzt die seit den 1950er-Jahren in ihrer Zahl stark zunehmenden verhaltenswissenschaftlichen Forschungsarbeiten erinnern daran, dass diese Perspektive zumindest ebenso wichtig ist wie eine klassisch betriebswirtschaftliche. In der Praxis wird die Integration des Wissens über Verhaltenswirkungen gleichsam zu einer wichtigen Dimension, die bei Gestaltung und Nutzung von Controllingsystemen zu beachten ist.

Andreas Wömpeners Dissertationsschrift ist motiviert durch diese Erkenntnis sowie durch die von ihm argumentierte fehlende Berücksichtigung der beschränkten Rationalität kognitiver Urteils- und Entscheidungsprozesse im Bereich der Unternehmenssteuerung. Solche Prozesse betreffen die Informationsverarbeitung, also zum Beispiel das Wahrnehmen, Schlussfolgern, Denken oder Problemlösen.

Vor diesem Hintergrund und der vom Autor vorgebrachten Forderung, betriebswirtschaftliche Konzepte an den Faktor "Mensch" anzupassen, liegt das Ziel der konzeptionellen Arbeit von Andreas Wömpener in zwei Dingen: In der Untersuchung der Auswirkungen beschränkter Rationalität von Organisationsmitgliedern auf das Instrument der Budgetierung und in der Ableitung von Ansatzpunkten zur Verbesserung der Budgetierungspraxis sowie der weiteren Forschung.
Nach einem kurzen problembegründenden Teil befasst sich Wömpener mit den Grundlagen des Themas, indem er im zweiten Kapitel auf die Budgetierung als zentrales Element der Unternehmenssteuerung eingeht. Zusätzlich leitet der Autor ein eigenes Phasenmodell der Budgetierung $\mathrm{ab}$, welches aus Planung, Kommunikation, Handlung, Kontrolle, Bewertung und Steuerung besteht.

Im dritten Kapitel zeigt der Autor die verschiedenen Facetten der Rationalität in der Literatur auf und vergleicht diese anschaulich miteinander. Besonders das für den Beitrag zentrale Konzept der beschränkten Rationalität findet hierbei Beachtung. Erwähnenswert ist zudem, dass sich die Arbeit auf das Nicht-Können der Handelnden z. B. in den Bereichen der Erwartung, Wahrnehmung, Kategorisierung oder Problemlösung konzentriert, dass also Aspekte des Nicht-Wollens, etwa durch mangelnde Motivation, in der Arbeit nicht betrachtet werden. Ferner verfolgt Wömpener bewusst einen Ansatz, bei dem er über das Individuum argumentiert und dadurch organisationale oder strukturelle Aspekte nicht thematisiert.

Den theoretischen Grundlagenteil bildet Kapitel vier, in welchem der Autor einen kurzen Überblick über verschiedene Theorien menschlichen Verhaltens gibt und sich somit hier und im Folgenden stark an der Schnittstelle zu anderen Disziplinen bewegt. Kern des Kapitels bildet die Ableitung eines Stimulus-Organismus-Reaktions-Modells (S-O-R-Modell), welches einen Stimulus, der über kognitive Strukturen und Prozesse (Organismus) eine Reaktion auslöst, abbildet.

Im Hauptteil der Arbeit werden zunächst aus der Literatur identifizierte Phänomene beschränkter Rationalität vorgestellt, $d$. $h$. solche, die dazu führen, dass eine Abkehr der Verfolgung eines Optimums und somit (lediglich) eine Satisfizierung eintritt. Dabei erfolgt eine grobe Dreiteilung in wahrnehmungs- (z. B. Opportunitätskosteneffekt) und bewertungsbedingte Phänomene ( $\mathrm{z}$. $\mathrm{B}$. Ankereffekt) sowie in solche, die auf einer fehlerhaften Selbst- oder Fremdeinschätzung (z. B. overconfidence bias) beruhen. In einer strukturierten Auflistung werden insgesamt siebzehn Phänomene kurz und mit leicht verständlichen Beispielen beschrieben und durch empirische Quellen belegt. Zusätzlich erfolgt jeweils eine verhaltenswissenschaftliche Einordnung anhand des vorab spezifizierten S-O-R-Modells. Außerdem wird die Bedeutung des Phänomens für die einzelnen Abschnitte des vorweg erarbeiteten Phasenmodells der Budgetierung herausgestellt.

Vor einer Zusammenfassung sowie einem Ausblick auf weiteren Forschungsbedarf führt Kapitel sechs die Ergebnisse des Hauptteils in einer komprimierten Form zusammen. In dieser Gesamtschau erreicht der Autor eine übersichtliche und teils priorisierende Struktur, die in dieser Form besonders dem praktisch orientierten Leser zur Verbesserung der Budgetierung dienlich sein kann. Beispielsweise könnten die Formalisierung von Abweichungsanalysen oder die Installation eines "kritischen Gegenspielers" bei der Bewertung und Steuerung objektivierend wirken und somit Rationalitätsdefizite abbauen.

In Summe lässt sich festhalten, dass Wömpener mit dieser Arbeit an vielen Stellen aufzeigt, wie wichtig es ist, die Verhaltenswirkungen von Controllingsystemen und insbesondere der Budgetierung auf Organisationsmitglieder zu überprüfen, um dysfunktionale Effekte auszuschließen.

Für Praktiker finden sich in dieser Schrift wohl besonders in Kapitel sechs Denkansätze, um einerseits eine verhaltensorientierte Sichtweise bei der Gestaltung bzw. der Verbesserung der Budgetierung zu berücksichtigen, und um sich andererseits der Verhaltenswirkungen ihrer Controllingsysteme bei deren Nutzung bewusst zu werden.

Wissenschaftlich interessierte Leser finden vor dem Hintergrund der Strukturierung durch die beiden für diese Arbeit abgeleiteten Modelle zur Budgetierung und Kognition eine Vielzahl von Hypothesen zur empirischen Überprüfung sowie eine strukturierte Übersicht der bisherigen Literatur.

Sebastian D. Becker, Vallendar 